\title{
SINGULAR SOLUTIONS FOR A CLASS OF GRUSIN TYPE OPERATORS
}

\author{
NICHOLAS HANGES AND A. ALEXANDROU HIMONAS \\ (Communicated by Jeffrey Rauch)
}

\begin{abstract}
We construct singular solutions for a one-parameter family of partial differential equations with double characteristics and with complex lower order terms. The parameter belongs to a discrete set which is described in terms of the spectrum of a related differential operator.
\end{abstract}

\section{INTRODUCTION}

Let $k$ be an odd positive integer, and for each $\lambda \in \mathbb{C}$ let $P_{\lambda}$ be the operator in $\mathbb{R}^{2}$ defined by

$$
P_{\lambda}=\partial_{x}^{2}+x^{2 k} \partial_{y}^{2}+i(\lambda+k) x^{k-1} \partial_{y}
$$

Also let $\Lambda_{k}$ be the set

$$
\Lambda_{k}=\{\lambda: \lambda=2 j(k+1) \text { or } \lambda=2 j(k+1)+2, j \in \mathbb{Z}\} .
$$

The local solvability of the operator $P_{\lambda}$ was studied by Gilioli and Treves [3] by using the method of concatenations. They proved that $P_{\lambda}$ is solvable if and only if $\lambda$ does not belong to the set $\Lambda_{k}$. The local hypoellipticity of a similar class of operators in an abstract setting was studied by Gilioli [2] by using concatenations different from the ones used in [3]. Earlier, and in the case $k=1$, Grusin [4] studied the hypoellipticity of $P_{\lambda}$ by using the spectral theory of the harmonic oscillator. Using its eigenvalues he described the discrete set $\Lambda_{1}$. The purpose of this work is to give an explicit computation of the set $\Lambda_{k}$ in terms of the eigenvalues of a generalized harmonic oscillator, and to present two methods of construction of singular solutions. One is by certain Fourier integrals with symbols expressed in terms of the eigenfunctions (see Theorem 1.1 and formula (2.1)). The other is by the method of concatenations of Gilioli and Treves [3] (see Theorem 3.1 and formula (3.14)). In particular we construct solutions with prescribed $C^{\infty}$ and analytic wave front sets. Next we state our first result:

Received by the editors October 14, 1994 and, in revised form, November 14, 1994.

1991 Mathematics Subject Classification. Primary 35H05.

Key words and phrases. Eigenvalue, eigenfunction, concatenations, hypoellipticity, double characteristics.

The first author was partially supported by NSF Grant DMS 91-04569.

The second author was partially supported by NSF Grant DMS 91-01161. 
Theorem 1.1. Let $k$ be an odd positive integer. If $\lambda \in \Lambda_{k}$, then the following hold:

1. There exists a $C^{\infty}$ solution to the equation $P_{\lambda} u=0$ which is not analytic.

2. For each $m \in \mathbb{N}_{0}=\{0,1,2, \ldots\}$ there exists a $C^{m}$ solution to the equation $P_{\lambda} u=0$ which is not $C^{m+1}$.

Remark. If $\lambda \in \Lambda_{k}$, then by using the same methods we can construct solutions $u$ to $P_{\lambda} u=0$ which are distributions of any finite order.

\section{Proof of Theorem 1.1}

Let $u(x, y)$ be defined by

$$
u(x, y)=\int_{0}^{\infty} e^{i \rho^{k+1} y} A(\rho x) w(\rho) d \rho,
$$

where the functions $A(x)$ and $w(\rho)$ are to be determined. We have

$\bar{P}_{\lambda} u(x, y)=\int_{0}^{\infty} e^{i \rho^{k+1} y} \rho^{2}\left[A^{\prime \prime}(\rho x)-x^{2 k} \rho^{2 k} A(\rho x)+(\lambda+k) x^{k-1} \rho^{k-1} A(\rho x)\right] w(\rho) d \rho$.

For $u$ to be a solution to $\bar{P}_{\lambda} u=0$, it suffices that $A$ satisfies the equation

$$
\left(-\frac{d^{2}}{d x^{2}}+x^{2 k}-(\lambda+k) x^{k-1}\right) A(x)=0 .
$$

In the next proposition we will state the spectral theory of the last equation on $\mathbb{R}$ with boundary conditions

$$
\lim _{|x| \rightarrow \infty} A(x)=0 .
$$

Let $L_{w_{k}}^{2}(\mathbb{R})$ be the Hilbert space of all functions $f: \mathbb{R} \rightarrow \mathbb{C}$ with the following inner product

$$
(f, g)_{w_{k}}=\int_{\mathbb{R}} f(x) \bar{g}(x) e^{-\frac{2}{k+1} x^{k+1}} x^{k-1} d x .
$$

Proposition 2.1. Let $k$ be an odd positive integer. Then for the boundary value problem (2.2) - (2.3) the following hold:

1. The set of eigenvalues is equal to

$$
k+\Lambda_{k}^{+}, \text {where } \Lambda_{k}^{+}=\left\{\lambda: \lambda=2 J(k+1), \text { or } \lambda=2[J(k+1)+1], J \in \mathbb{N}_{0}\right\} .
$$

2. If an eigenvalue is of the form $2 J(k+1)+k$ for some $J \in \mathbb{N}_{0}$, then the corresponding eigenspace is generated by the even function

$$
B_{k, J}^{0}(x) e^{-\frac{1}{k+1} x^{k+1}}, \text { with } B_{k, J}^{0}(x)=\sum_{j=0}^{J} b_{j(k+1)} x^{j(k+1)},
$$

where for $0 \leq j \leq J$

$b_{j(k+1)}=(-1)^{J+j}(k+1)^{J} 2^{j}\left(\begin{array}{l}J \\ j\end{array}\right)[J(k+1)-1][(J-1)(k+1)-1] \ldots[(j+1)(k+1)-1]$. 
If an eigenvalue is of the form $2[J(k+1)+1]+k$ for some $J \in \mathbb{N}_{0}$, then the corresponding eigenspace is generated by the odd function

$$
B_{k, J}^{1}(x) e^{-\frac{1}{k+1} x^{k+1}}, \text { with } B_{k, J}^{1}(x)=\sum_{j=0}^{J} b_{j(k+1)+1} x^{j(k+1)+1},
$$

where for $0 \leq j \leq J$

$b_{j(k+1)+1}=(-1)^{J+j}(k+1)^{J} 2^{j}\left(\begin{array}{l}J \\ j\end{array}\right)[J(k+1)+1][(J-1)(k+1)+1] \ldots[(j+1)(k+1)+1]$.

3. The set of generalized Hermite polynomials $\left\{B_{k, J}^{\ell}\right\}_{J \in \mathbb{N}_{0}}^{\ell=0,1}$ is an orthogonal basis for the Hilbert space $L_{w_{k}}^{2}(\mathbb{R})$.

Remark. If $k=1$, then $1+\Lambda_{1}=\left\{2 J+1, J \in \mathbb{N}_{0}\right\}$ is the set of eigenvalues for the classical harmonic oscillator. Also, $B_{1, J}^{0}$ are the even Hermite polynomials, and $B_{1, J}^{1}$ are the odd Hermite polynomials.

The set $\Lambda_{k}$ appears naturally as an expression of the eigenvalues of this problem. The singular solutions are integral expressions of the corresponding eigenfunctions.

The proof of Proposition 2.1 can be obtained by transforming equation (2.2) to an equation satisfied by the orthogonal polynomials corresponding to the weight function $e^{-x^{2}}|x|^{2 \kappa}$, for $\kappa=\frac{1}{2} \frac{k-1}{k+1}$. Then one is reduced to Problem 25 in Szegö's book [11]. Here we shall give a direct and self-contained proof of Proposition 2.1. The eigenfunctions are computed explicitly and are shown to be an orthogonal basis. These facts, although not needed here, are of independent interest.

Proof of 1 . In this case we choose $w(\rho)=e^{-\rho}$. Then for $\lambda \in \Lambda_{k}^{+}$by Proposition 2.1 there exists a function $A \in \mathcal{S}(\mathbb{R})$, with $A(0) \neq 0$ or $A^{\prime}(0) \neq 0$, satisfying equation (2.2). Since $A \in \mathcal{S}\left(\mathbb{R}^{n}\right),(2.1)$ defines a $C^{\infty}$ solution to the equation $P_{\lambda} \bar{u}=0$. Without loss of generality we assume that $A(0) \neq 0$. Since

$$
\begin{aligned}
\partial_{y}^{j} u(0,0) & =i^{j} A(0) \int_{0}^{\infty} \rho^{j(k+1)} e^{-\rho} d \rho \\
& =i^{j} A(0)[j(k+1)] !,
\end{aligned}
$$

we conclude that $\bar{u}$ is not real analytic in $\mathbb{R}$.

Next we apply $P_{\lambda}$ to $u(x, y)$ in (2.1) and we see that $P_{\lambda} u=0$ if $A$ satisfies the equation

$$
\left(-\frac{d^{2}}{d x^{2}}+x^{2 k}+(\lambda+k) x^{k-1}\right) A(x)=0 .
$$

Since $\lambda+k=-[(-\lambda-2 k)+k]$, by Proposition 2.1 the last equation has a solution $A \in \mathcal{S}(\mathbb{R})-0$ if $-\lambda-2 k \in \Lambda_{k}^{+}$, which is equivalent to

$$
\lambda \in\{\lambda: \lambda=2 j(k+1) \text { or } \lambda=2 j(k+1)+2, \quad j=-1,-2, \ldots\} .
$$

Then, as before, $u$ is a $C^{\infty}$ but not an analytic solution to equation $P_{\lambda} u=0$.

Proof of 2. It follows by choosing $w(\rho)=\left(1+\rho^{k+1}\right)^{N}$, for an appropriate $N=$ $N(m)<0$. Also for any $m \in \mathbb{N}_{0}$, by choosing an appropriate $N=N(m)>0$, we can construct a solution to $P_{\lambda} u=0$ which is a distribution of order $m$. 
The proof of Theorem 1.1 will be complete if we prove Proposition 2.1.

Proof of Proposition 2.1. First we make the following substitution:

$$
A(x)=e^{-\frac{1}{k+1} x^{k+1}} B(x) .
$$

Then equation (2.2) takes the form

$$
-B^{\prime \prime}+2 x^{k} B^{\prime}-\lambda x^{k-1} B=0 .
$$

If $k=1$, then (2.8) becomes the Hermite equation.

If in equation (2.8) we let

$$
B(x)=\sum_{j=0}^{\infty} b_{j} x^{j},
$$

then the coefficients $b_{j}$ satisfy the relations

$$
b_{j+2}=0, \text { for } 0 \leq j \leq k-2 \text {, and } b_{j+k+1}=\frac{2 j-\lambda}{(j+k)(j+k+1)} b_{j}, j=0,1,2, \ldots
$$

We shall need the following lemma.

Lemma 2.1. For a fixed $\lambda \in \mathbb{R}$ let $B_{k, \lambda}^{0}$ be the solution to equation (2.8) with initial conditions

$$
B_{k, \lambda}^{0}(0)=1 \text { and } \frac{d B_{k, \lambda}^{0}}{d x}(0)=0,
$$

and let $B_{k, \lambda}^{1}$ be the solution of equation (2.8) with initial conditions

$$
B_{k, \lambda}^{1}(0)=0 \text { and } \frac{d B_{k, \lambda}^{1}}{d x}(0)=1 .
$$

Then $B_{k, \lambda}^{0}$ is a polynomial if and only if

$$
\lambda=2 J(k+1), \text { for some } J \in \mathbb{N}_{0},
$$

and $B_{k, \lambda}^{1}$ is a polynomial if and only if

$$
\lambda=2 J(k+1)+2, \quad J \in \mathbb{N}_{0} .
$$

Proof. By (2.10) and (2.11) we obtain $b_{j}=0$, if $j \neq J(k+1)$ for some $J \in \mathbb{N}_{0}$, and

$$
b_{0}=1 \text { and } b_{(j+1)(k+1)}=\frac{2 j(k+1)-\lambda}{[j(k+1)+k](j+1)(k+1)} b_{j(k+1)}, j \in \mathbb{N}_{0} .
$$

Also by (2.10) and (2.12) we obtain $b_{j}=0$, if $j \neq J(k+1)+1$ for some $J \in \mathbb{N}_{0}$, and

$$
b_{1}=1, b_{(j+1)(k+1)+1}=\frac{2[j(k+1)+1]-\lambda}{(j+1)(k+1)[(j+1)(k+1)+1]} b_{j(k+1)+1}, j \in \mathbb{N}_{0} .
$$

Then Lemma 2.1 follows from (2.15) and (2.16).

For $\lambda$ as in equalities (2.13) or (2.14) the corresponding $B_{k, \lambda}^{0} e^{-x^{k+1} /(k+1)}$ and $B_{k, \lambda}^{1} e^{-x^{k+1} /(k+1)}$ decay exponentially fast near $\pm \infty$. In the next lemma we will show that this is not the case for all the other $\lambda$. 
Lemma 2.2. Let $\alpha$ be a real number such that $\frac{1}{k+1}<\alpha<\frac{2}{k+1}$. We have the following:

If $\lambda \neq 2 J(k+1), J \in \mathbb{N}_{0}$, then there exist $C_{\lambda}>0$ and $R_{\lambda}>0$ such that

$$
\left|B_{k, \lambda}^{0}(x) e^{-\frac{1}{k+1} x^{k+1}}\right| \geq C_{\lambda} e^{\left(\alpha-\frac{1}{k+1}\right) x^{k+1}},|x| \geq R_{\lambda} .
$$

If $\lambda \neq 2 J(k+1)+2, J \in \mathbb{N}_{0}$, then there exist $C_{\lambda}>0$ and $R_{\lambda}>0$ such that

$$
\left|B_{k, \lambda}^{1}(x) e^{-\frac{1}{k+1} x^{k+1}}\right| \geq C_{\lambda} e^{\left(\alpha-\frac{1}{k+1}\right) x^{k+1}},|x| \geq R_{\lambda} .
$$

If $\lambda \notin \Lambda_{k}^{+}$, then every non-zero solution $A$ to equation (1.1) satisfies the estimate

$$
|A(x)| \geq C e^{\left(\alpha-\frac{1}{k+1}\right) x^{k+1}}
$$

near at least one of $+\infty$ or $-\infty$, where $C$ depends on $A$.

Proof. It follows by comparing the coefficients of $B_{k, \lambda}^{l}$ given by (2.15) and (2.16) with the coefficients of the exponential $e^{\alpha x^{k+1}}$.

Lemmas 2.1 and 2.2 give the first two parts of Proposition 2.1. The even polynomials are computed by letting in $(2.15) \lambda=2 J(k+1)$ for some $J \in \mathbb{N}_{0}$. The odd polynomials are computed by letting in $(2.16) \lambda=2 J(k+1)+2$ for $J \in \mathbb{N}_{0}$.

Now we prove the last part of Proposition 2.1.

Proof of part 3 in Proposition 2.1. A short computation yields the orthogonality. Next we shall show the completeness. Let $f$ be a function in $L_{w_{k}}^{2}(\mathbb{R})$ such that

$$
\int_{\mathbb{R}} f(x) B_{k, J}^{\ell}(x) e^{-\frac{2}{k+1} x^{k+1}} x^{k-1} d x=0,
$$

for all $\ell \in\{0,1\}$ and $J \in \mathbb{N}_{0}$. We will show that $f=0$ a.e. in $\mathbb{R}$. By (2.17) and the form of the $B_{k, J}^{\ell}$ we obtain the relations:

$$
\begin{aligned}
& \int_{\mathbb{R}} f(x) p\left(x^{k+1}\right) e^{-\frac{2}{k+1} x^{k+1}} x^{k-1} d x=0, p \in \mathcal{P}, \\
& \int_{\mathbb{R}} f(x) x p\left(x^{k+1}\right) e^{-\frac{2}{k+1} x^{k+1}} x^{k-1} d x=0, p \in \mathcal{P},
\end{aligned}
$$

where $\mathcal{P}$ is the set of all polynomials in one variable.

By the Cauchy-Schwarz inequality we obtain that if $f \in L_{w_{k}}^{2}(\mathbb{R})$, then

$$
f(x) e^{|\xi||x|^{(k+1) / 2}} e^{-\frac{2}{k+1} x^{k+1}} x^{k-1} \in L^{1}(\mathbb{R})
$$

for every $\xi \in \mathbb{R}$. If we write $\cos \left(\xi x^{(k+1) / 2}\right)$ as a series and use $(2.20)$, then by the dominated convergence theorem we can pass the integral inside the sum and by also using (2.18) we obtain

$$
\int_{\mathbb{R}} \cos \left(\xi x^{(k+1) / 2}\right) f(x) e^{-\frac{2}{k+1} x^{k+1}} x^{k-1} d x=0, \xi \in \mathbb{R} .
$$


By the last relation and the change of variables $t=x^{\frac{k+1}{2}}$ we obtain that

$$
\int_{0}^{\infty} \cos (\xi t) f_{E}\left(t^{\frac{2}{k+1}}\right) e^{-\frac{2}{k+1} t^{2}} t^{\frac{k-1}{k+1}} d t=0, \xi \in \mathbb{R}
$$

where $f_{E}(x)=\frac{1}{2}[f(x)+f(-x)]$. By $(2.21)$ and the Fourier inversion theorem we obtain

$$
f_{E}\left(t^{\frac{2}{k+1}}\right) e^{-\frac{2}{k+1} t^{2}} t^{\frac{k-1}{k+1}}=0 \text { a.e. } t \in(0, \infty),
$$

which implies that

$$
f(x)+f(-x)=0 \text { a.e. for } x \in \mathbb{R} .
$$

Similarly by using (2.19) we show that

$$
x f(x)-x f(-x)=0 \text { a.e. for } x \in \mathbb{R} .
$$

By using (2.22) and (2.23) we obtain that $f=0$ a.e. in $\mathbb{R}$. This completes the proof of Proposition 2.1.

\section{The Method of CONCATENATions}

Let the vector field $L$ in $\mathbb{R}^{2}$ be defined by

$$
L=\partial_{x}+i x^{k} \partial_{y}
$$

Then the operator $P_{\lambda}$ in (1.1) can be written as

$$
P_{\lambda}=\bar{L} L+i \lambda x^{k-1} \partial_{y} .
$$

Also for $\mu \in \mathbb{R}$ let

$$
V_{\mu}=x L+\mu .
$$

Following Gilioli and Treves [3] we have the following concatenation formulas.

Lemma 3.1. For any $\lambda$ in $\mathbb{R}$ we have:

$$
\bar{V}_{\frac{\lambda}{2}+k+2} P_{\lambda}=P_{\lambda+2(k+1)} \bar{V}_{\frac{\lambda}{2}+k}
$$

and

$$
V_{-\frac{\lambda}{2}+2} P_{\lambda}=P_{\lambda-2(k+1)} V_{-\frac{\lambda}{2}} .
$$

Also simple computations yield the additional formulas

$$
x P_{2}=\bar{L}(x L-1)=\bar{L} V_{-1}
$$

and

$$
x P_{-2 k-2}=L(x \bar{L}-1)=L \bar{V}_{-1} .
$$

Next we shall use the method of concatenations to construct singular solutions to the operator $P_{\lambda}$ with prescribed microlocal singularities. 
Theorem 3.1. Let $P_{\lambda}$ be the operator (1.1) and $\gamma_{0}=\left(0, y_{0} ; 0, \eta_{0}\right), \eta_{0} \neq 0, a$ characteristic point of $P_{\lambda}$. Then the following hold:

(1) $P_{\lambda}$ is not hypoelliptic, or analytic hypoelliptic, at $\gamma_{0}$, with $\eta_{0}<0$, if $\lambda \in \Lambda_{k}^{+}$.

(2) $P_{\lambda}$ is not hypoelliptic, or analytic hypoelliptic, at $\gamma_{0}$, with $\eta_{0}>0$, if $\lambda \in \Lambda_{k}^{-}$. Here $\Lambda_{k}^{+}$is as in (2.4) and $\Lambda_{k}^{-}=\Lambda_{k}-\Lambda_{k}^{+}$, where $\Lambda_{k}$ is defined in (1.2).

Proof of (1). Let $\gamma_{0}$ be a characteristic point with $\eta_{0}<0$. First we observe that the function

$$
Z(x, y)=y-\frac{i}{k+1} x^{k+1}
$$

is a solution to $L Z=0$. In addition if we define

$$
u_{0}(x, y)=(Z(x, y))^{m+\frac{1}{2}}, m \in \mathbb{N},
$$

then $u$ is a $C^{m}$ solution to equation $L u=0$ which does not belong to $C^{m+1}$. In particular $u$ is singular at $\gamma_{0}$. Since

$$
P_{0} u_{0}=\bar{L} L u_{0}=0,
$$

$u$ is a solution to $P_{0} u=0$ which is singular at $\gamma_{0}$. Next we will show how starting from $u_{0}$ we can construct a singular solution at $\gamma_{0}$ for $P_{\lambda}, \lambda=2 j(k+1), j \in \mathbb{N}_{0}$. Suppose that a distribution $u$, which is singular at $\gamma_{0}$, is a solution to the equation

$$
P_{2 j(k+1)} u=0 .
$$

Then by (3.11) and concatenation formula (3.4) applied for $\lambda=2 j(k+1)$ we obtain

$$
P_{2(j+1)(k+1)} w=0, \text { where } w=\bar{V}_{j(k+1)+k} u .
$$

Then by the following lemma we obtain that $w$ is singular at $\gamma_{0}$.

Lemma 3.2. Let $\mu \in \mathbb{N}$ and $u \in \mathcal{D}^{\prime}\left(\mathbb{R}^{2}\right)$. If $P_{\lambda} u$ is regular at $\gamma_{0}$, with $\eta_{0}<0$, for some $\lambda \in \mathbb{R}$, and $\bar{V}_{\mu} u$ is regular at $\gamma_{0}$, then $u$ is regular at $\gamma_{0}$.

Proof of Lemma 3.2. If $\bar{V}_{\mu} u$ is regular at $\gamma_{0}$, then $x^{\mu-1} \bar{V}_{\mu} u$ is also regular at $\gamma_{0}$. By applying the formula

$$
\bar{L}\left(x^{\mu} u\right)=x^{\mu-1} \bar{V}_{\mu} u,
$$

we obtain that $\bar{L}\left(x^{\mu} u\right)$ is regular at $\gamma_{0}$. Since $\bar{L}$ is hypoelliptic for $\eta_{0}<0$ (see Hörmander [7], vol. IV, p. 84), we obtain that $x^{\mu} u$ is regular at $\gamma_{0}$. Now by using the assumption that $P_{\lambda} u$ is regular at $\gamma_{0}$, which implies additional regularity on $u$, we obtain that $u$ is regular at $\gamma_{0}$ (see Hörmander [7], vol. I, p. 267). This completes the proof of Lemma 3.2.

To summarize, so far we have shown that if $u_{0}$ is a singular solution of $P_{0}$ at $\gamma_{0}$, say $u_{0}$ is given by (3.9), then

$$
u_{2(j+1)(k+1)}=\bar{V}_{j(k+1)+k} \bar{V}_{(j-1)(k+1)+k} \cdots \bar{V}_{k} u_{0}
$$


is a singular solution to $P_{2(j+1)(k+1)} u=0$. What remains now is the construction of singular solutions at $\gamma_{0}$ for $\lambda=2 j(k+1)+2$. For this we first show that $P_{2}$ is not hypoelliptic at $\gamma_{0}$. Let

$$
w_{0}=x u_{0} .
$$

Then we obtain that

$$
P_{2} w_{0}=0 \text { and } w_{0} \text { is singular at } \gamma_{0} .
$$

Now by starting with (3.16) and by applying concatenation formula (3.4) we construct singular solutions at $\gamma_{0}$ to $P_{\lambda} u=0$ for all $\lambda=2 j(k+1)+2, j \in \mathbb{N}_{0}$. The formula of the singular solutions is very similar to (3.14) with $u_{0}$ replaced by $w_{0}$ given by (3.15).

Proof of (2). It is analogous to the proof of (1). We start with a singular solution to $\bar{L} u=0$ and then by using concatenation formula (3.5) we construct singular solutions at $\gamma_{0}$ with $\eta_{0}>0$, to $P_{\lambda} u=0, \lambda \in \Lambda_{k}^{-}$. This completes the proof of Theorem 3.1.

Remark. By using the method of concatenations we can also construct a $C^{\infty}$ solution to $P_{\lambda} u=0$ with a prescribed analytic wave front set. All we need to do is to start with a function $h$ which is holomorphic for $\operatorname{Im} z<0$ or $\operatorname{Im} z>0$, and $C^{\infty}$ up to $\operatorname{Im} z=0$, and then replace $u_{0}$ in $(3.9)$ by $u_{0}=h(Z)$ or $u_{0}=h(\bar{Z})$.

In a forthcoming article we will show that the necessary conditions of Theorem 3.1 for hypoellipticity and analytic hypoellipticity of $P_{\lambda}$ are also sufficient. The $P_{\lambda}$ will be models for a wide class of non-transversally elliptic operators with double characteristics. Such operators exhibit discrete phenomena in analogy with classic work on transversally elliptic operators of Grusin [4], Boutet de Monvel - Treves [1] and Hörmander [5]. The work of these authors stands in contrast to the work on operators with real first order term which do not exhibit discrete phenomena (e.g. Hörmander [6], Kohn [8], Oleinik - Radkevic [9] and Rothschild - Stein [10]).

\section{REFERENCES}

1. L. Boutet de Monvel and F. Treves, On a class of pseudodifferential equations with double characteristics, Inventiones Math. 24 (1974), 1-34. MR 50:5550

2. A. Gilioli, A class of second-order evolution equations with double characteristics, Ann. Scuola Norm. Sup. Pisa Cl. Sci. 4, no. 3 (1976), 187-229. MR 55:10882

3. A. Gilioli and F. Treves, An example in the local solvability theory of linear PDE's, American Journal of Math. 24, No. 2 (1974), 366-384. MR 50:7761

4. V. V. Grusin, On a class of hypoelliptic operators, Math. Sbornik. 83, No. 125 (1970), 456-473. MR 43:5158

5. L. Hörmander, A class of hypoelliptic pseudo-differential operators with double characteristics, Math. Ann. 217 (1975), 165-188. MR 51:13774

6. L. Hörmander, Hypoelliptic second order differential equations, Acta Math. 119 (1967), 147171. MR 36:5526

7. L. Hörmander, The Analysis of Linear Partial Differential Operators, vol. I-IV, SpringerVerlag, Grundlehren der Mathematischen Wissenschften, 1984. MR 87d:35002b

8. J.J. Kohn, Pseudo-differential operators and hypoellipticity, Proceedings of symposia in pure mathematics XXIII (1973), 61-70. MR 49:3356

9. O. A. Oleinik and E. V. Radkevic, Second order equations with nonnegative characteristic form, AMS and Plenum Press, 1973. MR 56:16112 
10. L.P. Rothschild and E.M. Stein, Hypoelliptic differential operators and nilpotent groups, Acta Math. 137 (1977), 247-320. MR 55:9171

11. G. Szegö, Orthogonal Polynomials, American Mathematical Society, 1959. MR 21:5029

Department of Mathematics, Herbert H. Lehman College-Cuny, Bronx, New York 10468-1589

E-mail address: nwhlc@cunyvm.cuny.edu

Department of Mathematics, University of Notre Dame, Notre Dame, Indiana 46556

E-mail address: Alex.A.Himonas.1@nd.edu 